\title{
Cognition-Aware Summarization of Photos Representing Events
}

\author{
Bei LIU ${ }^{\dagger a)}$, Nonmember, Makoto P. KATO ${ }^{\dagger}$, Member, and Katsumi TANAKA ${ }^{\dagger}$, Nonmember
}

\begin{abstract}
SUMMARY The use of photo summarization technology to summarize a photo collection is often oriented to users who own the photo collection. However, people's interest in sharing photos with others highlights the importance of cognition-aware summarization of photos by which viewers can easily recognize the exact event those photos represent. In this research, we address the problem of cognition-aware summarization of photos representing events, and propose to solve this problem and to improve the perceptual quality of a photo set by proactively preventing misrecognization that a photo set might bring. Three types of neighbor events that can possibly cause misrecognizations are discussed in this paper, namely sub-events, super-events and sibling-events. We analyze the reasons for these misrecognitions and then propose three criteria to prevent from them. A combination of the criteria is used to generate summarization of photos that can represent an event with several photos. Our approach was empirically demonstrated with photos from Flickr by utilizing their visual features and related tags. The results indicated the effectiveness of our proposed methods in comparison with a baseline method.

key words: cognition-aware, photo summarization, event
\end{abstract}

\section{Introduction}

With the popularization of social networks and photosharing communities such as Facebook, Twitter, Flickr, Pinterest, and Instagram, photos are becoming a more common way to express and share our daily life with friends. In addition to their informativeness and visual appeal, photos play an important role as an online communication tool. For example, a user can post several photos to let his/her followers know about a trip taken to Japan in summer with a description written in Chinese. As the saying goes, A picture is worth a thousand words, and his/her friends can quickly grasp the user's experience even without reading the description carefully, and can save time spent in social network communities, which continues to increase year after year. Moreover, even non-Chinese speakers can understand a Chinese user's updates only by looking at the posted photos. As seen in the example above, photo-based communication has advantages in terms of its efficiency (less time necessary to understand the content), perspicuity (able to view more content at the same time), and language-independence (comprehensible for speakers of any language), although it cannot be correctly understood unless appropriate photos are used for the communication. Thus, the core technology

\footnotetext{
Manuscript received February 18, 2016.

Manuscript revised August 2, 2016.

Manuscript publicized September 1, 2016.

${ }^{\dagger}$ The authors are with the Department of Social Informatics, Kyoto University, Kyoto-shi, 606-8501 Japan.

a) E-mail: liubei@dl.kuis.kyoto-u.ac.jp

DOI: 10.1587/transinf.2016EDP7079
}

that enables efficient photo-based communication is to automatically generate a cognition-aware summary of photos that can be accurately recognized by other users.

In this paper, we propose a way of tackling the problem of generating a cognition-aware summary of photos for a given event. Cognition-aware summaries of photos for an event are defined as those with high perceptual quality for the event, which is defined as the accuracy and quickness to recognize an event by the summaries. For the example of "traveling to Japan", we expect as output photos that might include "shinkansen bullet train", "old Japanese temples", and "the user wearing a kimono". In contrast, a photo summary is not considered to be cognition-aware if it contains photos that cannot be recognized as "traveling to Japan", for example, "cars in Japan" and "Japanese ships" (since their appearance is not much different from those in other countries). As we discussed earlier, a cognition-aware summary of photos can enable language-independent, efficient communication in a social network and has many applications including event-driven photo thumbnails for a personal photo collection and a communication tool in multilingual communities.

Two main differences exist between our research and existing work on photo summarization. First of all, the purpose of our work is to improve the perceptual quality of a photo set, which differs from that of existing work, which is to extract an informative overview of a photo collection [1], [2]. It follows that our approach also differs from the approach used in photo summarization. For example, Sinha et al. [3] addressed the problem of summarizing personal photos from life events and aimed to best represent a photo collection with its small representative subset. However, such a photo set does not necessarily have high perceptual quality, as their approach does not consider other similar events in which a resulting photo set can be misrecognized. Suppose that a user wants to summarize photos taken during his/her trip to the Kansai area in Japan. When the summary consists of sights in Kyoto, Japanese food in Osaka, a shinkansen train in Kobe, and women wearing kimono in Nara, one can easily misrecognize the summary as "trip to Kyoto" since photos of Japanese food, shinkansen trains, and women in kimono would also be common in photos of Kyoto. While the summary does not include any specific photos of the Kansai area, it will be misrecognized as a "trip to Japan" instead. From the viewpoint of perceptual quality, the photo set should be generated by taking into account the event's neighbor event (e.g., "trip to Kyoto", "trip 
to Japan") and minimizing possible misrecognitions (e.g., including photos of landmarks in several cities in Kansai). Therefore, we analyze the relationship between events and summarize possible neighbor events that can easily cause misrecognition. Then we propose a method to prevent users from misrecognizing a photo set as neighbor events.

Three types of neighbor events are defined for those events that might cause misrecognition: sub-events, superevents and sibling-events. We study the reasons for these misrecognitions and then put forth three criteria to minimize the misrecognitions, namely, sub-event coverage, superevent coverage, and difference from sibling events. A greedy algorithm is applied to integration of three criteria for generating approximately optimal photo set with high perceptual quality.

Experiments were conducted using a considerable number of photos from Flickr, and the experimental results showed that our proposed method was able to achieve high perceptual quality in comparison with a baseline method, although the quality highly depends on the queries used and size of the generated photo sets. The results also indicated that photo summarization technologies are not always effective for generating cognition-aware summarization of photos.

Three contributions of this paper are briefly described:

1. We raise the problem of cognition-aware summarization of photos for a given event from the standpoint of viewers who expect to find important points and events from photos, which is different from the problem addressed in existing research on photo summarization (Sects. 1 and 2),

2. We propose a way to solve the problem by using the method of generating a photo set with high perceptual quality by minimizing possible misrecognitions, namely, sub-event, super-event and sibling-event misrecognition (Sects. 3 and 4), and

3. We explain the effectiveness of implementing our approach in experiments using a large number of photos from Flickr. The results were evaluated and compared with a baseline method based on an existing photo summarization method (Sect. 6).

We present a conclusion in Sect. 7 .

\section{Related Work}

This research concerns two main problems: event representation and photo summarization. We review here other studies related to these two problems and point out the differences between them and our work.

\subsection{Event Representation}

Event representation has been explored in several different study fields for years. Language and cognition have opened up the field of how the mind deals with the experience of events [4]. As in computer science, there are studies focusing on how to detect and represent events in natural language, and with the evolution of social networking systems (SNSs), some studies have been done on processing social events [5], [6]. In the multimedia domain, event detection has been widely researched with both videos and photos. Different approaches have been proposed to understand the structure of complex events in videos [7], [8].

In recent years, a lot of research has been done on the prediction of events from photos. Chen and Roy [1] proposed an approach to detect Flickr photos depicting events. Brenner and Izquierdo [9] incorporated different features to detect social events from collaboratively annotated photo collections. These studies solve the problem of detecting events and clustering photos while not considering the characteristics of events and the relationship between events. In this paper, we focus more on how to select cognition-aware photos to represent an event by paying attention to the concerned events' relations.

\subsection{Photo Summarization}

Most research on photo summarization has focused on personal photo collections. Platt et al. [10] presented an overview of a user's photo collections generated by an image clustering algorithm that considered time and color. Other features such as time, location [11], social context features (such as tags and comments) [12], and blog posts [13] were utilized to help with photo summarization.

Unsupervised approaches were proposed in [14] for event clustering based on time and image content. An event-clustering algorithm was developed to automatically segment photos into events and sub-events for albuming based on date/time information and color content of the photos [15]. PageRank was employed to mine the most informative information from photos of the same event in [16].

The metrics of a good photo summarization differ. Sinha and Jain [12] proposed generating a summary on the basis of three properties: relevance, diversity, and coverage. While Wen and Lin [17] focused on two metrics: representativeness and diversity.

The goal of this work is different from the above studies on photo summarization. We focus more on the accurate recognition of a specific event from our generated photo set, which is emphasized on finding cognition-aware photos.

\section{Preliminaries}

In this section, we will first define the events that we focused on and then explain the problem we addressed in this research.

\subsection{Events}

A common definition of an event is "a segment of time at a given location that is conceived by an observer to have a beginning and an end" [18]. Many studies have focused on 
specific events such as current news items, sports events or earthquakes [1], [19], [20].

In this research, we are tackling real life events, which are normal events related to our everyday life, for example, a "football game". We refer to some existing research about general events [21], and define events in this work as:

Definition 1 (Event): An event involves human activities and can be specified with time and location.

An event can be denoted with certain terms, e.g., "travel Japan" and "hiking summer", where the terms "travel" and "hiking" represent activities, while "Japan" and "summer" specify the location and time of each event.

Note that many terms can imply an activity depicted in photos, including terms in verb or noun form, since everything in the photo can reflect what a photo taker is doing while taking that photo. For example, the term "lavender" indicates a plant and cannot be regarded as an activity in a common sense. However, if a photo depicts lavender, it means the photo is taken when a user is viewing the lavender. As a result, the term "lavender" also indicates the activity of "viewing lavender" by taking the activity of the photo taker into consideration. Therefore, in this research, the terms describing the activities involved in an event are not restricted to verbs when the event is shown with photos.

\subsection{Problem Definition}

The problem we tackle in this work can be defined as:

Definition 2 (Event Representation Problem): Given an event $e \in E$, a collection of photos $P$, and the size of an output photo set $n$, return photo summarization with a photo set $S_{e} \subset P_{e}$ of size $n$ that maximizes perceptual quality,

where $E$ represents all possible events, and $P_{e}$ refers to photos that are related to event $e$ among the whole photo dataset $P$. Perceptual quality is defined as:

Definition 3 (Perceptual Quality): Perceptual quality of a photo set for an event is the metric that measures the accuracy and quickness of the photo set being recognized as the event.

Higher perceptual quality makes a photo set easier to recognize as a certain event.

Photo collection $P$ is a large set of photos that were taken by different users, while $P_{e}$ can be photos of event $e$ taken by many users as well as one user. Users can decide an appropriate size of the output photo set depending on their application, such as thumbnails and cover photos for a personal photo library or SNS posts. As a matter of fact, current SNSs also limit the number of photos a user can upload for a new update. For example, Twitter allows four photos for each tweet, and Weibo (Chinese twitter) permits up to nine photos for each tweet. Although Facebook can accept more photos for one update, it can display around five photos as a thumbnail for each update. Therefore, a small photo set is preferred, especially within the size of ten or fewer photos.
Thus, we aim to produce a cognition-aware photo set representing events with photo set size of fewer than nine photos, which is often used as an upper size limit when combining photos using photo editing softwares.

For each event $e \in E$, there are many neighbor events that may cause misrecognitions.

Definition 4 (Neighbor Events): Neighbor events of an event are events that are similar to the event and can easily cause misrecognitions.

The definition of misrecognition is as follows:

Definition 5 (Misrecognition): Misrecognition happens when something (such as an event) is recognized as something else.

In order to find a photo set with high perceptual quality, we should avoid neighbor events that will cause misrecognition.

\section{Approach}

In this section, we introduce our approach to generate a photo set with high perceptual quality.

\subsection{Overview}

As we mentioned in Sect. 1, our goal is to find a photo set that represents an event with high perceptual quality so that users can recognize that exact event after viewing the photo set. We introduce an assumption that:

Assumption 1: A photo set that is not likely to be misrecognized as another event is likely to be recognized accurately.

With this assumption, we propose a method to minimize misrecognitions from neighbor events that can easily cause misunderstandings and to accordingly achieve high perceptual quality. Sub-event coverage, super-event coverage, and the difference from sibling events are used as quantized criteria, and they are proposed to prevent three misrecognitions.

A combination of the three criteria is used to make the objective function, and a greedy algorithm is applied to generate an approximate optimal photo set.

We also propose to generate neighbor events by considering the relationship between events. Visual features, including global and local features, and social features such as tags attached to photos are employed in our approach.

\subsection{Misrecognition Situations}

There are three types of misrecognitions that can occur when a user looks at a photo set and tries to determine which event it is, and they are mainly caused by three types of corresponding neighbor events:

1. The first kind is sub-event misrecognition, which means users recognize a photo set of an event as a subevent of the original event. 
Definition 6 (Sub-Events): Event A is a sub-event of event B if A can only represent part of B.

For example, the event "travel" consists of several sub-events such as "transport" (actually it is "travel transport", but we removed "travel" for simplification), "shopping", and "sightseeing". Given the event "travel" to be recognized, a photo set that includes only photos of "transport" will cover one single sub-event of "travel". This photo set has a high possibility of being recognized as "travel transport". Intuitively, we can avoid sub-event misrecognitions by generating a photo set that covers as many sub-events as possible.

2. The second situation is called super-event misrecognition, which means a photo set is thought of a superevent of the original event.

Definition 7 (Super-Events): Event B is a super-event of event A if only part of B can be represented by A.

Super-events correspond to sub-events. When a photo set consists of only photos that are common in its super-event, super-event misrecognition will occur. For instance, super-events of "travel in Kyoto" are "Kyoto" (all the events that can happen in Kyoto) and "travel". Moreover, photos of "sakura", "temple", and "food" are often taken for the event "travel in Kyoto". However, they can also represent the event of "Kyoto", which refers to all the activities that may happen in Kyoto. If a photo set only includes photos of Kyoto but does not include any photos portraying travel, people prefer to treat it as its super-event "Kyoto" when they cannot identify more specific content. A simple solution to avoid this type of misrecognition is to cover all possible super-events within a photo set.

3. Sibling-event misrecognition is the last type of misrecognition, and it indicates the case where a user misrecognizes a photo set of an event as its sibling-event.

Definition 8 (Sibling-Events): Event $\mathrm{A}$ is a sibling event of event B if A and B can both represent different parts of the same super-event.

For example, a photo set for "conference party" includes photos of "people communicating", "dishes and desserts", and "proposing a toast", and all of them can be recognized as "conference party". However, without labels indicating "conference party", it will be difficult for a user to tell what kind of party the set is about. Users may take it to be a "birthday party" if a photo includes cakes. "Birthday party" and "conference party" have the super-event "party", so each of them presents a specific part of the event "party". We can avoid this kind of misrecognitions by using a photo that does not imply a specific kind of sibling-events, such as a photo of people gathering rather than a photo of a cake in this example.
(A)

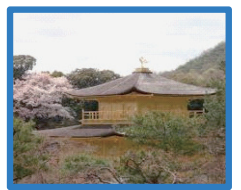

(1) Kyoto

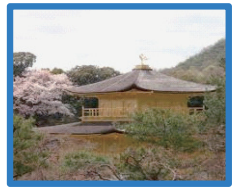

(4) Kyoto

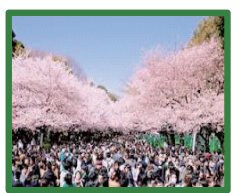

(2) Tokyo

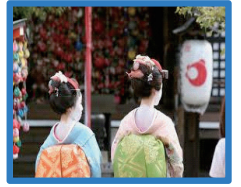

(5) Kyoto

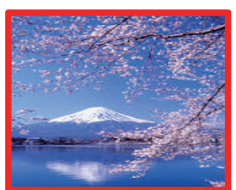

(3) Fuji

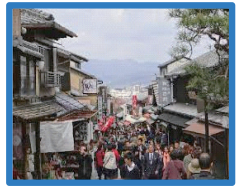

(6) Kyoto
Fig. 1 Example of Sub-Event Coverage: photo set with only photos of "Kyoto" (B) will indicate "Kyoto travel spring Japan", which is a sub-event of "Japan spring travel", while a photo set that covers different parts of Japan (A) will not have this problem.

\subsection{Sub-Event Coverage}

In order to avoid the first type of misrecognition-sub-event misrecognition-we propose the following assumption:

A photo set that covers only a single or a few sub-events may cause sub-event misrecognitions.

Under this assumption, an ideal photo set should cover as many sub-events as possible. By using the example we used in Sect. 4.2, a photo set that contains "transport", "food", and "landscape" would be better to represent the event "travel" than one that only contains "transport". Thus, the sub-event coverage is used to evaluate how likely a photo set can prevent sub-event misrecognitions.

Figure 1 also shows an example of sub-event coverage. Photo set (B) covers only photos of traveling in Kyoto, which is just one part of travel in Japan, and users might easily perceive that the photos represent "Kyoto travel in spring" rather than "Japan travel in spring", and this is a sub-event misrecognition. In contrast, if a photo set, for example (A), covers photos of travel in different parts of Japan such as Kyoto, Tokyo and Mt. Fuji, users will not be misled about the specific place, and the correct position "Japan" can be easily ascertained.

The sub-event coverage can be measured by borrowing an idea in search result diversification, which aims to retrieve search results that cover as many topics as possible in response to a given query [22]. The approach used in search result diversification is to estimate the probability that all the topics will be covered with at least one search result, and to find a set of search results that maximizes this probability. Therefore, as with search result diversification, we estimate the probability that all the sub-events will be covered with at least one photo and try to find a set of photos that maximizes this probability. Thus, the sub-event coverage $\operatorname{SubCov}(S, e)$ is defined as follows:

$$
\begin{aligned}
& \operatorname{SubCov}(S, e) \\
& =\sum_{v \in \operatorname{Sub}(e)} P(v \mid e)\left(1-\prod_{s \in S} P(c=0 \mid s, v)\right)
\end{aligned}
$$

where $e$ is a given event, $S$ is a photo set, $\operatorname{Sub}(e)$ refers to 
sub-events of event $e, P(v \mid e)$ is the probability that event $e$ contains sub-event $v$ as well, and $P(c=0 \mid s, v)$ is the probability that photo $s$ does not present sub-event $v$ with $c$ (binary value) to indicate whether it is presented (value 1 ) or not (value 0 ). We assume a unique distribution for $P(v \mid e)$ due to the lack of prior knowledge for this probability, i.e., $P(v \mid e)=\frac{1}{|\operatorname{Sub}(e)|}$.

An intuitive interpretation of this formula is that $\operatorname{SubCov}(S, e)$ becomes high if at least one of the photos in a photo set $S$ has high probability $P(c=1 \mid s, v)$ for all the sub-events of $e$.

Below, we discuss a method of estimating the probability $P(c=1 \mid s, v)$, which is a complement of $P(c=0 \mid s, v)$, $P(c=1 \mid s, v)=1-P(c=0 \mid s, v)$. A basic assumption here is that photo $s$ is likely to cover sub-event $v$ if $s$ is similar to photos that were taken in sub-event $v$. We used $k$-nearest neighbor distance $\mathrm{k}-\mathrm{NND}(s, v)$, which is the average distance of $k$-nearest neighbor photos of photo $s$ in photo set $P_{v}$, to measure the similarity between photo $s$ and photos of event $v$ [23]. In addition to its simplicity, the computation of the $k$-nearest neighbor distance is efficient, since $k$-nearest neighbor search has been extensively studied in the literature. We obtain the following formula by taking the inverted distance of $\mathrm{k}-\mathrm{NND}(s, v)$ with an exponential function:

$$
P(c=1 \mid s, v)=\exp (-\lambda \cdot \mathrm{k}-\mathrm{NND}(s, v))
$$

where $\lambda$ is used to control the shape of this distribution.

In summary, sub-event coverage $\operatorname{SubCov}(S, e)$ measures how likely a photo set can prevent sub-event misrecognitions. With a higher $\operatorname{SubCov}(S, e)$ value, photo set $S$ is able to cover more sub-events of event $e$. A photo set with high sub-event coverage is expected to avoid sub-event misrecognitions and consequently achieve high perceptual quality.

\subsection{Super-Event Coverage}

Super-event misrecognition can happen when photos in a photo set are only related to part of the original event's super-events and cannot cover all of them. This observation led to an assumption that:

A photo set that covers only one or a few super-events may cause super-event misrecognitions.

A photo set $S$ that can prevent this type of misrecognition covers all the super-events $\operatorname{Sup}(e)$ of event $e$ with at least one photo in the photo set $S$. In the example of photo set (A) in Fig. 2, when "travel" and "Kyoto" are both emphasized in the photo set, it will be easier to recognize both events and form the event "travel in Kyoto". Thus, we apply the algorithm of sub-event coverage in Eq. (1) to each super-event and compute the super-event coverage of original event $\operatorname{SupCov}(S, e)$, as described in Eq. (3).

$$
\begin{aligned}
& \operatorname{Sup} \operatorname{Cov}(S, e) \\
& =\sum_{u \in \operatorname{Sup}(e)} P(u \mid e)\left(1-\prod_{s \in S} P(c=0 \mid s, u)\right)
\end{aligned}
$$

Only if a photo set covers all super-events will it
(A)

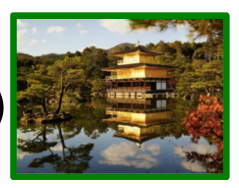

(1) Kyoto

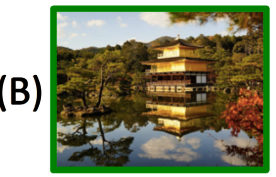

(4) Kyoto

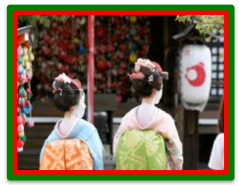

(2) Kyoto, Travel

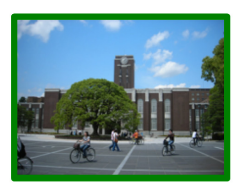

(5) Kyoto

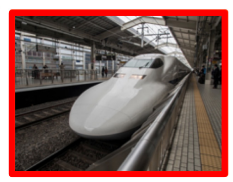

(3) Travel

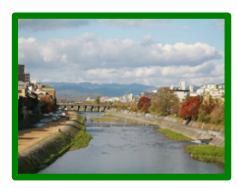

(6) Kyoto
Fig. 2 Example of Super-Event Coverage: photo set with only photos of "Kyoto" (B) will indicate "Kyoto", which is a super-event of "Kyoto travel", while a photo set that covers both "Kyoto" and "travel" (A) will not have this problem.

achieve high super-event coverage. For example, a photo set with high super-event coverage for the event "travel in Kyoto" would be one that has high sub-event coverage for both the events "travel" and "Kyoto". Higher super-event coverage guarantees that all super-events are covered by a photo set.

\subsection{Difference from Sibling-Event}

To avoid sibling-event misrecognition, we should not use photos that present only a few sibling-events.

A photo similar to just a few sibling-events can cause sibling-event misrecognitions.

Thus, photos that are similar to all sibling-events or not similar to any sibling-event under one super-event are preferable in order to avoid sibling-event misrecognition. With photos that are similar to all sibling-events under one super-event, our consideration is that all the sibling-events should contain common features of the same super-event. For example, to find photos of "travel Japan", a photo that is common in all sub-events of "travel" is generally sufficient to avoid sibling-event misrecognition. However, since super-event coverage guarantees that all the super-events are covered in the output photo set, there is no necessity to use the idea again in preventing sibling-event misrecognition. To this point, we propose a difference from sibling-events to avoid misrecognition from sibling-events. The formula is as follows:

$$
\operatorname{SibDif}(S, e)=1-\prod_{s \in S} \max _{v \in \operatorname{Sib}(e)} P(c=1 \mid s, v)
$$

where $\operatorname{Sib}(e)$ are all sibling-events of $e$ under all its superevents. The distribution for $P(c=1 \mid s, v)$ is assumed to be equal, just as in Sect.4.3, which measures the probability that photo $s$ covers event $v$. For each photo in a photo set, we hope that the maximum possibility of covering siblingevents is as small as possible. Note that this criterion is used to complement sub-event coverage and super-event coverage.

As a result, photos that are different from all sibling- 


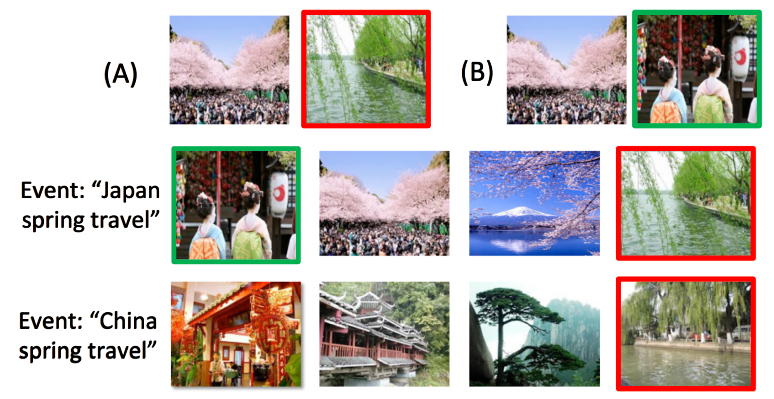

Fig. 3 Example of difference from sibling-events. Photos of willow trees are common in both Japan and China in spring, so a photo set that includes a photo of a willow tree (A) is not as good as one that includes a photo of women in kimono (B), since a photo of women in kimono is unique to Japan and will not cause misrecognition.

events will rank higher. In the example in Fig. 3, willow trees are quite common in both "spring in China" and "spring in Japan", while they are not as common in other places; thus, photos of willow trees are often taken in spring in these two places. If we use a photo of a willow tree to represent the event "Japan spring travel", users cannot decide where it is and may think it is in China. Instead, photos of women wearing kimono are most likely to be taken in Japan, and it is much less common for other places to have similar photos. Thus, it will achieve a higher score than a photo of willow trees in consideration of sibling-events of "Japan spring travel".

\subsection{Photo Set Generation}

By combining three criteria to avoid the three types of misrecognition, we generate one objective function to measure to what extent a photo set can minimize misrecognitions. Sub-event coverage, super-event coverage, and the difference from sibling-events are combined in order to maximized the objective function $f(S, e)$.

$$
\begin{aligned}
& f(S, e) \\
& =\alpha \operatorname{SubCov}(S, e)+\beta \operatorname{Sup} \operatorname{Cov}(S, e)+\gamma \operatorname{SibDif}(S, e)
\end{aligned}
$$

where $\alpha, \beta$, and $\gamma$ are parameters that determine which criteria should be emphasized.

Our objective can now be reformulated as a problem of finding a photo set of size $n$ for a given event $e$ that maximizes the objective function $f(S, e)$. Unfortunately, finding an optimal photo set is an NP-hard (Non-deterministic Polynomial-time hard) problem.

Lemma 1: $f(S, e)$ is NP-hard.

When photos can belong to multiple photo sets, there may not exist a single ordering of photo sets such that the objective function of $f(S, e)$ is maximized for all possible $S$. The reason is that a set of photos optimal for $f\left(S^{\prime}, e\right)$, where $\left|S^{\prime}\right|=n-1$, need not be a subset of the optimal value of $f(S, e)$, where $|S|=n$.

Because the set function $f(S, e)$ is monotonic and submodular (see the Appendix), we can apply the greedy al-

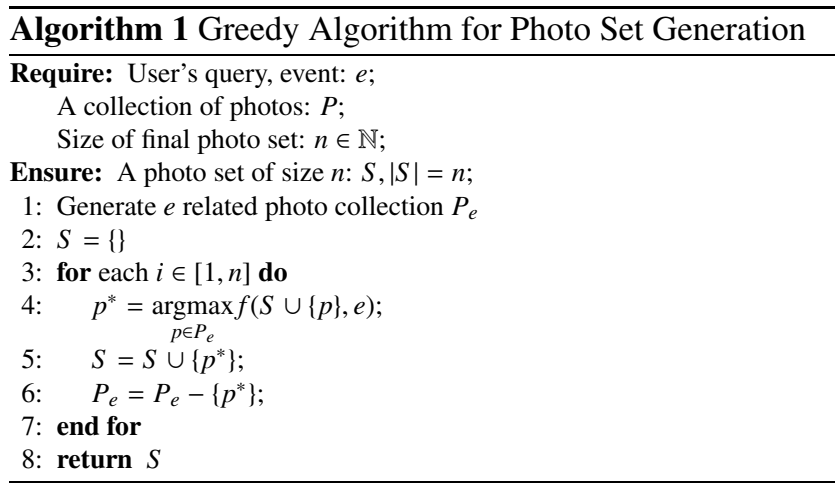

gorithm and guarantee that the result returns a $(1-1 / e)$ approximation of the maximum [24], which often gives a good approximation to the optimum. We start with an empty photo set $S$ and iteratively add a photo $p \in P_{e}$ to $S$ that maximizes $f(S \cup\{p\}, e)$ until the size of the photo set $|S|$ reaches $n$. The greedy algorithm is described in Algorithm 1.

\section{Neighbor Event Generation}

To find proper neighbor events that can easily cause misrecognition, we utilize photos of events and their surrounding social information such as tags.

\subsection{Generating Sub-Events}

According to the definition, sub-events are events that can present part of an event. We need to find ones that can easily cause misrecognition. Our strategy of generating sub-events is to add a keyword to the keyword set of the original event and to let the added keyword specify a certain part of that event. As a result, our objective becomes finding keywords to be added and in particular, keywords that can be easily confused with the target event $e$. Added keywords are selected from tags of event $T_{e}$ if they comply with two criteria of selected sub-events: they are relevant (representative and visually similar) and irredundant.

A sub-event needs to be representative to event $e$ because representative content is likely to remind viewers of event $e$. For example, "travel Kyoto temple" is a representative sub-event of the event "travel Kyoto", and photos of "travel Kyoto temple" have a high possibility to be regarded as "travel Kyoto". Term frequency-inverted document frequency (TF-IDF) is used to determine the importance of representative sub-events. We first compute TF (in $P_{e}$ )-IDF of all event-related tags $T_{e}$ [25]. A tag is denoted by $t$, and $\mathrm{tf}-\mathrm{idf}(t, e)$ represents its TF-IDF value.

Photos of a sub-event that could cause misrecognition are usually visually similar to photos of the event. We calculate the visual similarity between $e$ and each sub-event, which is defined by the photo similarity of events $e$ and $t$, VisualSim $(t, e)$. Visual similarity between two events is obtained by measuring the Euclidean Distance between their visual features [26]. Suppose we have two events $e_{1}$ and $e_{2}$ : 
photos of them are denoted by $P_{e_{1}}$ and $P_{e_{2}}$. Visual features of each tag are obtained by taking the average visual features of all photos in $P_{e_{1}}$ and $P_{e_{2}}$. Visual features of each tag are also obtained from global visual features such as color (RGB and HSV), and local features with a 1000-D bag of visual words [27].

The relevance of a tag $t$ to event $e$ is the harmonic mean of its TF-IDF and visual similarity value:

$$
\operatorname{SubRel}(t, e)=\frac{2 \operatorname{tf}-\operatorname{idf}(t, e) \operatorname{VisualSim}(t, e)}{\operatorname{tf}-\operatorname{idf}(t, e)+\operatorname{VisualSim}(t, e)}
$$

To ensure that all selected sub-events are efficient and are able to cover many different aspects of the event, we need to reduce the redundancy of resulting sub-events. Here, redundant sub-events refer to ones that describe almost the same content. For instance, images of "spring Japan" and "spring Nihon" are basically the same. We use context similarity between tags as a diversity metric, since tags that often appear together with the same vector of tags are supposed to present the same content [28]. Context similarity, ContextSim(t1, t2), measures whether two tags are similar by considering their neighbor tags. For example, "cloud" and "sky" are often tagged with the same set of tags in one photo, such as "blue, water, tree", and are therefore very similar based on the tag context.

Then maximal marginal relevance (MMR) [29] is applied to find tags that balance relevance with $e$ and diversity from selected sub-event tag set $W_{e} \subset T_{e}$, as shown in the following formula:

$$
\begin{aligned}
& \operatorname{MMRSub}(t, e) \\
& =\underset{t_{i} \in T_{e} \backslash W_{e}}{\operatorname{argmax}}\left[\theta \operatorname{SubRel}\left(t_{i}, e\right)-(1-\theta) \max _{t_{j} \in W_{e}} \operatorname{ContextSim}\left(t_{i}, t_{j}\right)\right]
\end{aligned}
$$

After finding several top sub-event tags with MMR, we generate sub-event $v$, whose keyword set is denoted as $K_{v}$, by adding each sub-event tag to the original event's keyword set $K_{e}$, i.e.,

$$
\operatorname{Sub}(e)=\left\{v \mid K_{v}=K_{e} \cup\{w\} \wedge w \in W_{e}\right\}
$$

Thus, if "cherry" and "Tokyo" are the top tags generated by our method of finding sub-events of "travel Japan", corresponding sub-events will be "cherry travel Japan" and "Tokyo travel Japan".

\subsection{Generating Super-Events}

In accordance with the idea of obtaining sub-events by adding one keyword to specify a certain part of an event, super-events are generated by removing each keyword from the original event's keyword set $K_{e}$, since subsets with a smaller number of keywords usually represent a more general concept than the original keyword set. Thus,

$$
\operatorname{Sup}(e)=\left\{u\left|K_{u} \subset K_{e} \wedge\right| K_{u}|=| K_{e} \mid-1\right\}
$$

where $K_{u}$ is a keyword set of super-event $u$. For example, super-events of "travel Japan" are "travel" and "Japan".

\subsection{Generating Sibling-Events}

Sibling-events present different parts of an event's superevent, and our objective is to find those that are likely to cause misrecognition. We produce sibling-events by specifying an event's super-events with one more word. Similar to the case of sub-event generation, the added word should satisfy the criteria of relevance (contextually and visually similar) and irredundancy. For each super-event $u$ of an event $e$, the absent word $k$ is one that generalizes the event, i.e., $k \in K_{e}-K_{u}$, $(u \in \operatorname{Sup}(e))$.

The added word should be contextually similar to the absent word in the context of a super-event, because words with a similar context in photos are often related to similar contents in the photos and will easily cause misrecognition. For example, "Kyoto" is similar to "Nara" in the context of the event "travel", since they are both related to the contents of "temple" and "traditional".

In addition, the added word should have a short distance with the absent word in regard to visual features since similar visual features can more easily cause misrecognition.

As a result, context similarity and visual similarity between words are combined to compute the relevance, and for this combination, we use their harmonic mean:

$$
\operatorname{SibRel}(t, k)=\frac{2 \text { ContextSim }(t, k) \operatorname{VisualSim}(t, k)}{\operatorname{ContextSim}(t, k)+\operatorname{VisualSim}(t, k)}
$$

With only relevance as the criterion to generate siblingevents, we find that there is some reduplication between sub-events and sibling-events. For example, "travel Japan Tokyo" is one of the top sub-events of "travel Japan" because many people who go to Japan for travel purposes will visit Tokyo (high TF value), and Tokyo travel is highly related to Japan travel, while it is not frequent in other events (high TF and IDF). However, we can understand that "travel Japan Tokyo" is almost the same event as "travel Tokyo", which is highly possible as a top sibling-event of "travel Japan" when relevance is the only criterion. In order to exclude sibling-events such as "travel Tokyo", we utilize the Jaccuard distance to measure the dissimilarity of new added word $t$ ("Tokyo") and abstract word $k$ ("Japan") in superevent $u$ ("travel") to ensure that sibling-events that are similar to sub-events are excluded. The Jaccard index is used to measure the concurrence of two words in an event by comparing the similarity and diversity of photos that contain each word as a tag. The tag Jaccard distance is the complementary to the Jaccard index of tags:

$$
\operatorname{TagJacDist}(t, k, u)=1-\frac{\left|P_{t+u} \cap P_{k+u}\right|}{\left|P_{t+u} \cup P_{k+u}\right|}
$$

A longer distance means two tags are not often tagged together in many photos of an event. Thus, they are better as sibling-events than those that are often tagged together, under the same condition that they are contextual and visually similar to each other. 
Table 1 Neighbor Events of "travel Japan spring"

\begin{tabular}{|c|c|c|}
\hline Method & Neighbor Event & Result \\
\hline Our Method & $\begin{array}{l}\text { Super-Event } \\
\text { Sibling-Event }\end{array}$ & $\begin{array}{l}\text { 'travel Japan spring park', 'travel Japan spring cherry', 'travel Japan spring Tokyo', 'travel Japan spring } \\
\text { tree', 'travel Japan spring flower', 'travel Japan spring sky', 'travel Japan spring Kyoto'... } \\
\text { 'travel Japan', 'travel spring', 'Japan spring' } \\
\text { 'travel Japan outside', 'travel Japan artistic', 'travel Japan beautiful', 'travel spring outdoor', 'travel spring } \\
\text { urban', 'travel spring traditional', 'Japan spring scenic', 'Japan spring cute', 'Japan spring sunlight'... }\end{array}$ \\
\hline TF-IDF & $\begin{array}{l}\text { Super-Event } \\
\text { Sibling-Event }\end{array}$ & $\begin{array}{l}\text { 'travel Japan spring flower', 'travel Japan spring landscape', 'travel Japan spring sky', 'travel Japan spring } \\
\text { tree', 'travel Japan spring water', 'travel Japan spring city', 'travel Japan spring light'... } \\
\text { 'travel Japan', 'travel spring', 'Japan spring' } \\
\text { 'travel Japan landscape', 'travel Japan sky', 'travel Japan city', 'travel spring flower', 'travel spring land- } \\
\text { scape', 'Japan spring flower', 'Japan spring tree', 'Japan spring Tokyo', 'Japan spring museum'... }\end{array}$ \\
\hline
\end{tabular}

Thus, the new relevance computation comes to:

$$
\begin{aligned}
& \operatorname{SibRel}(t, k, u) \\
& =\frac{2 \operatorname{ContextSim}(t, k) \operatorname{VisualSim}(t+u, k+u)}{\operatorname{ContextSim}(t, k, u)+\operatorname{VisualSim}(t+u, k+u)} \cdot \operatorname{TagJacDist}(t, k, u)
\end{aligned}
$$

by multiplying the original relevance by the Jaccard tag distance. In this case, "travel China" will be ranked higher than "travel Tokyo" when finding sibling-events of "travel Japan" under the super-event "travel".

Semantic similarity, SemanticSim $\left(t_{1}, t_{2}\right)$, is used for diversity because tags with the same meaning should be avoided. We implement it by using path similarity, which computes the semantic relatedness of words by counting the number of nodes along the shortest path between words in the "is-a" hierarchies of Wordnet [30]. Let $W_{u} \subset T_{u}$ be the current sibling-event tag set under one of the super-events $u$. The target function of the MMR algorithm is as follows:

$$
\begin{aligned}
& \operatorname{MMRSib}(t, u, e) \\
& =\underset{t_{i} \in T_{u} \backslash W_{u}}{\operatorname{argmax}}\left[\phi \operatorname{SibRel}\left(t_{i}, k\right)-(1-\phi) \max _{t_{j} \in W_{u}} \operatorname{SemanticSim}\left(t_{i}, t_{j}\right)\right]
\end{aligned}
$$

With several top tags $w$ with MMR $W_{u}$ of each superevent $u$, we obtain sibling events $v$ of event $e$ under $u$ so that the keyword set of $v$ is the result of adding $w$ to $u$ 's keyword set, i.e.

$$
\operatorname{Sib}(e, u)=\left\{v \mid K_{v}=K_{u} \cup\{w\} \wedge w \in W_{u}\right\}
$$

As we have described, these methods to create neighbor events are based on our requirement to find neighbor events that can easily cause misrecognition. We will show the effectiveness of our method compared with the baseline method (TF-IDF) in the next section.

\section{Experiments}

To evaluate the performance of our approach to avoid misrecognition as well as to evaluate the effectiveness of generating neighbor events, we conducted some experiments by using photos crawled from Flickr. We assessed the performance of neighbor event generation and photo set generation separately.

\begin{tabular}{|c|c|c|c|}
\hline ID & Event & ID & Event \\
\hline 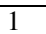 & birthday party & 26 & winter london \\
\hline 2 & bungee jump & 27 & award ceremony \\
\hline 3 & car accident & 28 & bar cocktail \\
\hline 4 & company meeting & 29 & bar concert \\
\hline 5 & conference party & 30 & beach wedding \\
\hline 6 & cook dinner & 31 & festival japan \\
\hline 7 & fashion show & 32 & island holiday \\
\hline 8 & football game & 33 & lavender france \\
\hline 9 & graduation ceremony & 34 & outdoor hotspring \\
\hline 10 & ice skating & 35 & paris shopping \\
\hline 11 & playing golf & 36 & rainbow mountain \\
\hline 12 & rock climbing & 37 & thunder city \\
\hline 13 & running on beach & 38 & travel hokkaido \\
\hline 14 & sports game & 39 & travel london \\
\hline 15 & sunset sea & 40 & yoga outdoor \\
\hline 16 & walk dog & 41 & boat autumn \\
\hline 17 & walk travel & 42 & christmas party \\
\hline 18 & wedding ceremony & 43 & concert night \\
\hline 19 & road trip summer & 44 & flight sunset \\
\hline 20 & summer hawaii & 45 & halloween costume \\
\hline 21 & sunrise mountain & 46 & morning walk \\
\hline 22 & swim beach summer & 47 & spring bike \\
\hline 23 & travel canada autumn & 48 & summer hiking \\
\hline 24 & travel japan spring & 49 & surf summer \\
\hline 25 & travel usa winter & 50 & thanksgiving dinner \\
\hline
\end{tabular}

The photo collection in our experiments consisted of more than 2.9 million photos crawled from Flickr, which
Table 250 events categorized based on whether the event is time-aware or location-aware

were used as the entire photo collection $P$.

\subsection{Generating Neighbor Events}

In this experiment, we tested 50 events (indicated in Table 2 . We picked these events from everyday life events by checking the frequently updated events in Flickr. Furthermore, the selected events are ones that have meaningful neighbor events (especially super-events based on our method). Neighbor events of these events were generated with our proposed method and a simple baseline method. In our method, parameters $\theta$ and $\phi$, which are used to balance the importance of similarity and diversity in generating sub-events and sibling-events, were set according to our preliminary experiments: $\theta=0.7, \phi=0.7$.

As for the baseline, we used TF-IDF to rank tags, and we generated sub-events by adding the top-ranked tags to a target event. Super-events were produced in the same way as in our method, while sibling-events were generated by using sub-events of these super-events with the TF-IDF method. 
Table 1 lists some examples generated with our method and the baseline method. From this table, we find that TFIDF always generates duplicate events such as "travel Japan landscape" and "travel spring landscape" for sibling-events. In addition, the resulting neighbor events are not necessarily ones that satisfy our definition of neighbor events. For example, "travel spring flower" is more appropriate as a subevent than a sibling-event.

However, neighbor events resulting from our method can meet the needs of neighbor event properties. For instance, "travel spring traditional" is a sibling-event of "travel Japan spring" under the super-event "travel spring". We can imagine that travelers who go to Japan in spring often visit some temples or shrines, which are all old buildings and represent traditional things. It is easy to mix up travel in Japan with travel in other traditional places. Let us take "travel Japan spring cherry" as another example. Japan is very famous for its cherry blossoms that only bloom in spring. Everyone who travels to Japan in spring will take photos of the cherry blossoms. Hence, it is a very important sub-event of "travel Japan spring", and our method can create it while the baseline method cannot.

Therefore, neighbor events generated by our method are closer to our expectation and correspond to reality much better. In particular, our proposed method was probably able to generate better sibling-events because we considered the absent term of each super-event. In contrast, the baseline method using TF-IDF generated many duplicate sibling-events probably due to the lack of consideration of the similarity between tags.

\subsection{Generating Photo Sets}

After obtaining neighbor events that can easily cause misrecognition, we utilized them in computing photo sets using the baseline method and our method separately and in combination. The perceptual quality of the generated photo sets was evaluated with a crowdsourcing service.

\subsubsection{Baseline}

VisualRank (VR) [31] was used as the baseline method to generate photo sets. VR is an algorithm that applies PageRank to photos, and it can be used to mine the most informative features from photos that belong to the same event [16]. The formula of VR is as follows: given $n$ photos, VR is recursively defined as

$$
\mathrm{VR}=d M^{*} \times \mathrm{VR}+(1-d) p
$$

where $p=\left[\frac{1}{n}\right]_{n \times 1} \cdot M^{*}$ is the column normalized adjacency matrix $M$, where $M_{i, j}$ is the similarity between photo $p_{i}$ and $p_{j}$, which is computed by the Euclidean distance between visual features of two photos. In order to guarantee that the resulting photo set does not include the same photos, we checked the visual similarity of each candidate photo and added photos to ensure their similarity Euclidean distance is

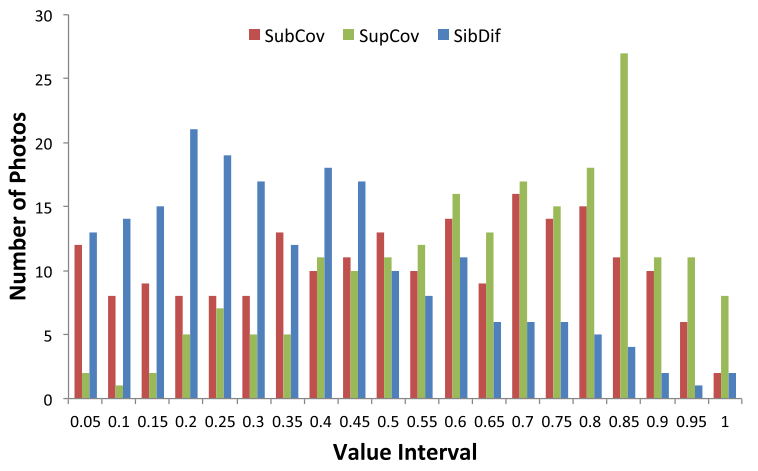

Fig. 4 Value distribution of three criteria for event "travel Japan spring".

greater than 0.1 .

\subsubsection{Setup}

We generated photo sets with the 50 events listed in Table 2 and used five sizes of photo sets for each event. Each of these events had more than 100 related photos from our photo collection. The average number of related photos for each event was 307.

We applied five methods, including the baseline VR method and four variations of our proposed method: an objective function with only sub-event coverage (SubCov), only super-event coverage (SupCov), only the difference from sibling-events (SibDif), and an objective function with a combination of three criteria (ALL), which maximizes the value of $f$ in Eq. (5). After getting the ranking score of event-related photos with these five methods, we used the top several photos to form a photo set.

We conducted preliminary experiments and checked the distribution of three types of scores for each candidate photo of the event "travel Japan spring" as displayed in Fig. 4. The result showed that normalized scores were equally distributed from 0 to 1 for three criteria, which enabled us to give the same parameter to each of the criteria and guarantee their equivalent importance in the target photo set. As a result, three parameters in the objective function were set as follows: $\alpha=1 / 3, \beta=1 / 3, \gamma=1 / 3$.

As mentioned in Sect. 3.2, a photo set is usually not larger than nine photos. For this reason, we used five sizes of photo sets, i.e. $n=1,3,5,7$, and 9. In total, there were 1250 photo set (event, method, size of photo set) combinations. The parameter $k$ of the $k$-nearest neighbor was set to 20 , and the parameter $\lambda$ was set to 25 in this experiment based on our preliminary test.

We used a crowdsourcing service to evaluate the perceptual quality of each photo set in two steps: labeling events to generated photo sets, and then obtaining the perceptual quality of a photo set by comparing the labeled event and the input event. Lancers ${ }^{\dagger}$, a crowdsourcing service in Japan, was used in our evaluation.

In the first task, five assessors were assigned to each

\footnotetext{
${ }^{\dagger}$ http://www.lancers.jp/
} 


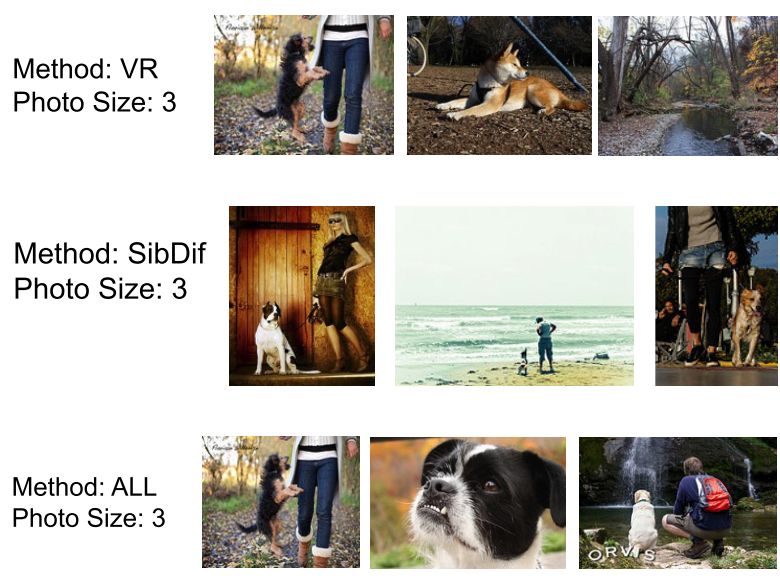

Fig. 5 Photo sets of event "walk dog" generated by different methods: VR (baseline VisualRank), SibDif (difference from sibling-events), and ALL (combination of three criteria).

photo set, and they were asked to label what they thought the photos represent. According to the previous definition, the perceptual quality of a photo set is better weighted by the accuracy and quickness of the user's perception of an event by looking at a photo set. In our experiment, we focused on the accuracy component by setting the quickness at a certain level while leaving the evaluation of quickness as an open issue due to practical difficulties. We asked the assessors to label what events they could recognize from a photo set in a few seconds, including the time, location, and activity.

The perceptual quality of a photo set was measured in the second step by evaluating the agreement between the input event of the photo set and the labels the assessors added.

Definition 9 (Estimated Perceptual Quality): Estimated perceptual quality of a photo set $S$ to represent an event $e$ is the average agreement between the event $e$ and events $E_{S}$, where $E_{S}$ denotes events that users can perceive from photo set $S$.

The agreement between two events was measured on a three-point scale: mismatch (score 0), partial match (score 1), and match (score 2). The assessor marked a label as a match if the label had the same meaning as the event, while the assessor gave a partial match to labels that partially overlapped keywords of the event. For example, "travel Japan" is a partial match, while "spring Japan travel" is a match to the event "travel Japan spring".

To guarantee high quality of the assessment, we added a dummy event-label pair every 9 pairs to make up one unit. The assessors gave scores in units and each of them could assign scores for up to 100 units. Scores marked by assessors who made a mistake on the dummy pair were excluded in the final result.

\subsubsection{Results}

Figure 5 shows photo sets of the event "walk dog" generated by the VR, SibDif and ALL methods. As we can see, VR

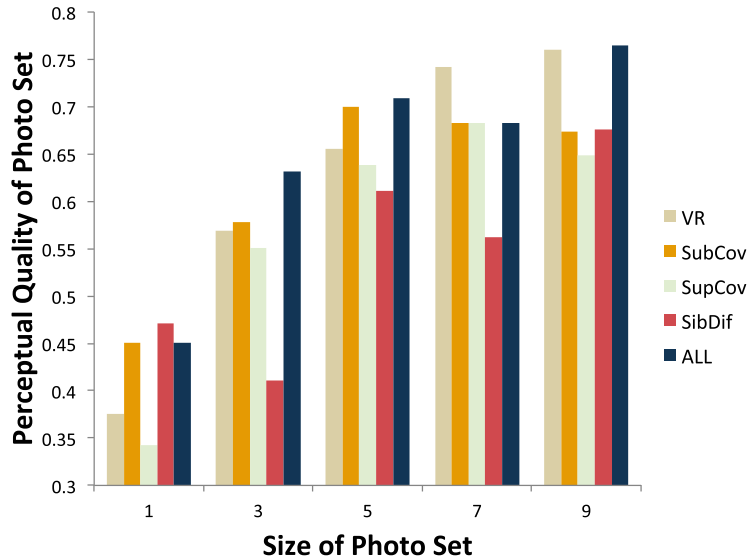

Fig. 6 Perceptual quality of five methods: we applied each method to 50 events in five different sizes of photo sets: $1,3,5,7$, and 9 .

gives very similar photos but fails to cover all sub-events such as "walk dog leash". It also lacks differentiation from sibling-events such as "raise dog" and "walk stroll". SibDif successfully avoids misrecognition from these siblingevents.

Figure 6 plots an overall comparison of the results of the five methods. The horizontal axis is the number of photos in the photo set, and the vertical axis is the average perceptual quality of each size, which is computed by the average score of agreement between target events and labeled events. The overall trend is clear: perceptual quality is gradually improved with more photos in a photo set. With more than five photos, the benefit of adding more photos begins to decrease.

We conducted a three-way ANOVA (Analysis of Variance) to test whether there was a difference between the effects of the methods, the sizes of a photo set, and the events and their interactions to the perceptual quality of a photo set. The ANOVA showed a significant difference in all of them: the type of method $(F(4,2500)=6.1, p<0.01)$, size of photo set $(F(4,2500)=51.26, p<0.01)$, and events $(F(49,2500)=35.5, p<0.01)$. Significant interactions were found among all combinations. These values demonstrated that the perceptual quality of a photo set varies with different sizes, different methods perform in various ways, and events can affect the performance.

From Fig. 6, we can also find that three criteria (SubCov, SupCov, and SibDif) show different performances with different photo set sizes. The performance of SubCov increases steadily until size five and stops rising, or even drops after adding more photos. This might be due to its coverage of good representative content of an event. With too many photos, high sub-event coverage will include photos with less important content, which confuses viewers.

SibDif is used as a supplementary criterion to avoid sibling-events. The result indicates that the performance is unstable. Photos that portray totally different events will also intuitively achieve a high score with SibDif, which results in atypical photos and lower perceptual quality. How- 


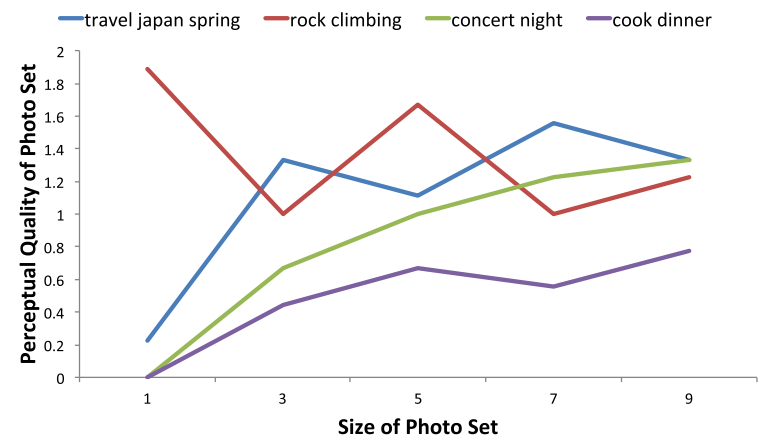

Fig. 7 Perceptual quality of several events in different sizes.

ever, it exhibits best performance with one photo. This may be attributed to the fact that it maximizes an event's difference from sibling-events that can cause misunderstandings, and users can easily figure out the right event without hesitation.

ALL performs best for most photo set sizes. It guarantees coverage of the important content of an event and maximizes the difference from similar neighbor events. Compared with the baseline method (VR), it demonstrates better performance with five or fewer photos. As discussed in the performance of sub-event coverage, having more photos can bring confusing and noisy information that may mislead viewers into perceiving it as another event. To check whether it is true with ALL method, we check four events and their performances of different sizes. As we can see from Fig. 7, events that contain a few scenes such as "rock climbing" can be easily recognized in a small photo set, while events that are more abstract and contain many different scenes gain better performance with more photos. Therefore, more photos do not necessarily convey accurate information, so a properly sized photo set is preferable.

In addition, we compared the final perceptual quality of photo sets for different neighbor events (generated by our proposed method and the TF-IDF method) to investigate whether the proposed neighbor events contribute to the final performance. The results are given in Table 3. Those numbers indicate that our proposed method improved the final performance of generating photo sets with higher perceptual quality by providing more suitable neighbor events.

In conclusion, our approach is able to generate photo sets of a proper size with higher perceptual quality compared with the baseline method.

\subsection{Analysis of Events and Perceptual Quality}

As indicated from the ANOVA, we can see that events play a role in affecting the performance of perceptual quality. To see the relation between a photo set's perceptual quality and event categories, especially regarding time and location, we classify all 50 events into four types based on whether the event is time-aware or location-aware.

The average perceptual quality of different types of events is shown in Fig. 8 for five methods. This figure re-
Table 3 Comparison of perceptual quality of photo sets generated by our proposed method and TF-IDF method.

\begin{tabular}{|c|c|c|c|}
\hline $\begin{array}{l}\text { Method of gen- } \\
\text { erating photo set }\end{array}$ & Size of photo set & 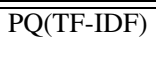 & 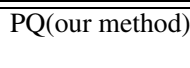 \\
\hline \multirow{5}{*}{ VR } & 1 & 0.31 & 0.38 \\
\hline & 3 & 0.51 & 0.57 \\
\hline & 5 & 0.54 & 0.66 \\
\hline & 7 & 0.62 & 0.74 \\
\hline & 9 & 0.61 & 0.76 \\
\hline \multirow{5}{*}{ SubCov } & 1 & 0.31 & 0.45 \\
\hline & 3 & 0.54 & 0.58 \\
\hline & 5 & 0.50 & 0.70 \\
\hline & 7 & 0.53 & 0.68 \\
\hline & 9 & 0.57 & 0.67 \\
\hline \multirow{5}{*}{ SupCov } & 1 & 0.30 & 0.34 \\
\hline & 3 & 0.56 & 0.55 \\
\hline & 5 & 0.58 & 0.64 \\
\hline & 7 & 0.55 & 0.68 \\
\hline & 9 & 0.57 & 0.65 \\
\hline \multirow{5}{*}{ SibDif } & 1 & 0.19 & 0.47 \\
\hline & 3 & 0.30 & 0.41 \\
\hline & 5 & 0.43 & 0.61 \\
\hline & 7 & 0.47 & 0.56 \\
\hline & 9 & 0.53 & 0.68 \\
\hline \multirow{5}{*}{ ALL } & 1 & 0.30 & 0.45 \\
\hline & 3 & 0.49 & 0.63 \\
\hline & 5 & 0.55 & 0.71 \\
\hline & 7 & 0.61 & 0.68 \\
\hline & 9 & 0.65 & 0.76 \\
\hline
\end{tabular}

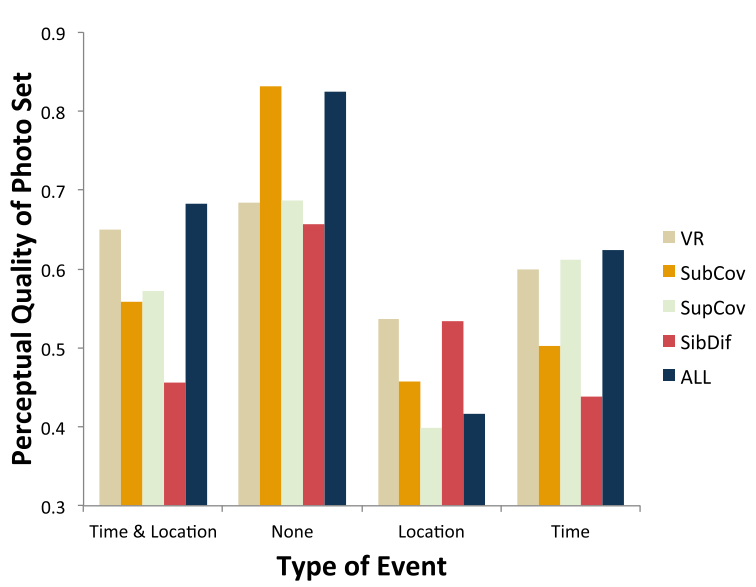

Fig. 8 Perceptual quality of different types of events and methods. Here, "None" corresponds to non-time specified or non-location specified, "time" to time-specified, "location" to location-specified, and "time \&location" to time specified and location specified.

veals that for all methods, perceptual quality is highest when time and location are not specified; in particular, SubCov reveals the best performance. Time is more easily represented than location with photos since the perceptual quality performance of most methods is higher with time-aware events than with location-aware events, except for SibDif. This result conforms to the fact that time is not easily confused because human beings hold almost the same perception about time, such as whether it is night or autumn. However, people see locations in different ways and might mix them up with other similar locations. That also explains why SibDif 


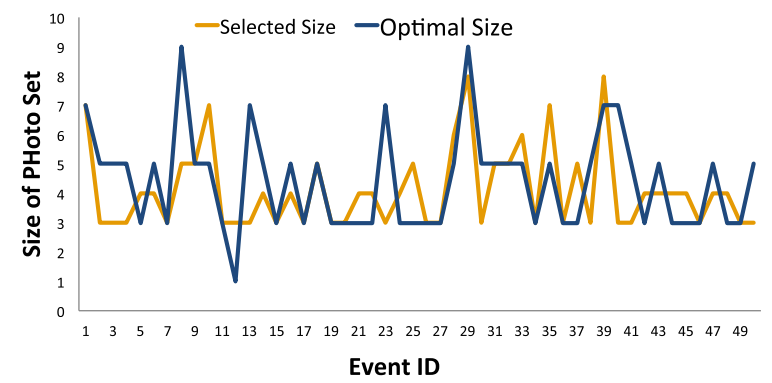

Fig.9 Evaluation of photo set size selection: sizes generated in automatically (yellow dots) and sizes selected manually by considering each event's perceptual quality and smallness of size(blue dots).

can better perform with location-aware events.

Moreover, ALL achieves high perceptual quality for most types of events except for location-specified ones. This result demonstrates that our approach fails in presenting location-aware events, although it can generate good cognition-aware photo sets for events in which time or location is not specified.

\subsection{Photo Set Size Selection}

In our experiment, the size of a photo set was given in advance. Now we propose a method to automatically determine the appropriate size of a photo set for a given event. The size was compared with sizes we set in the previous experiment by balancing perceptual quality and small sizes.

We can see from the comparison of perceptual quality at different sizes that the benefit of adding another photo begins to decrease after a certain size, which means we can determine the appropriate size by finding the point where perceptual quality and smallness of the photo set size is balanced. Our approach is as follows; in every step of the greedy algorithm shown in Algorithm 1, we check the objective function value and compare it with previous values. If the benefit of the added photo is smaller than our required threshold $\sigma$, the iteration will end, and previous photos will be returned as a result.

Let $S_{k-1}$ and $S_{k}$ be the result of the $(k-1)$-th and $k$-th iteration during the process of the greedy algorithm. When another photo $p$ is added to photo set $S_{k}$ in the $(k+1)$-th iteration, the following condition should be satisfied:

$$
\frac{f\left(S_{k} \cup\{p\}, e\right)-f\left(S_{k}, e\right)}{f\left(S_{k}, e\right)-f\left(S_{k-1}, e\right)} \geqslant \sigma
$$

Intuitively, we will stop the iteration of the greedy algorithm if the relative gain in perceptual quality by adding one more photo is small.

With the 50 events, we conducted an experiment with our proposed method and generated the best size for each event. Here, we set the threshold $\sigma=0.5$. The size automatically computed by our method is called the selected size. We also select a size from five sizes $(1,3,5,7,9)$ in the photo set generation experiment that has a relatively high perceptual quality for a small size and call it the optimal size. A comparison of these two sizes is shown in Fig. 9.
From this result, we can see that the general trend between selected size and optimal size is very similar. It demonstrates that our method can give an approximate size by balancing both perceptual quality and size smallness.

\section{Conclusion and Future Work}

In this research, we first proposed a method to achieve cognition-aware summarization of photos presenting events from the perspective of viewers rather than photo takers. To improve the perceptual quality of a photo set, which is defined as the accuracy and quickness of a user's recognition of an event from the photo set, we focused on preventing misrecognition of neighbor events, which is different from the work in traditional research on photo sets. The reasons for highly possible misrecognitions were analyzed, and three criteria were raised to measure the degree of preventing them. A greedy algorithm was then applied to generate photo sets by maximizing the objective function that combines the three criteria. We compared the performance of VisualRank and our approach. The results showed that our proposed approach improved the perceptual quality in different sizes of photo sets.

Moreover, we analyzed the relationship between the perceptual quality of photo sets and event types considering time and location. The results indicated that time is easier to express with photos than location. Additionally, events that are not time- or location-specified can be better perceived in photos by users. We also conducted an experiment to find the optimal sizes for photo sets of events using our proposed method, and the resulting photo set sizes were compared with manually selected optimal sizes. We found that our method was able to give an approximate optimal size under the premise of high perceptual quality and small photo set size.

Additional work is needed to address this problem more comprehensively. Although we have considered different factors of an event in the analysis, for example, time and location, we did not make them distinct when generating neighbor events, which will be part of our future work. Moreover, these factors should be taken into consideration when analyzing the relationships between events.

\section{References}

[1] L. Chen and A. Roy, "Event detection from flickr data through wavelet-based spatial analysis," Proc. 18th Conf. Information and Knowledge Management, CIKM '09, pp.523-532, ACM, 2009.

[2] P. Sinha, S. Mehrotra, and R. Jain, "Summarization of personal photologs using multidimensional content and context," Proc. 1st Int. Conf. Multimedia Retrieval, ICMR '11, pp.4:1-4:8, ACM, 2011.

[3] P. Sinha and R. Jain, "Extractive summarization of personal photos from life events," Proc. 2011 Int. Conf. Multimedia and Expo, ICME '11, pp.1-6, IEEE, 2011.

[4] J. Bohnemeyer and E. Pederson, Event representation in language and cognition, Cambridge University Press, 2011.

[5] A. Agarwal and O. Rambow, "Automatic detection and classification of social events," Proc. 2010 Conf. Empirical Methods in Natural Language Processing, EMNLP '10, pp.1024-1034, 2010. 
[6] D.-D. Nguyen, M.-S. Dao, and T.-V.T. Nguyen, "Natural language processing for social event classification," Proc. 6th Int. Conf. Knowledge and Systems Engineering, vol.326, pp.79-91, 2015.

[7] K. Tang, L. Fei-Fei, and D. Koller, "Learning latent temporal structure for complex event detection," Proc. Conf. Computer Vision and Pattern Recognition, pp.1250-1257, IEEE, 2012.

[8] Z. Ma, Y. Yang, Z. Xu, S. Yan, N. Sebe, and A.G. Hauptmann, "Complex event detection via multi-source video attributes," Proc. Conf. Computer Vision and Pattern Recognition, pp.2627-2633, IEEE, 2013.

[9] M. Brenner and E. Izquierdo, "Social event detection and retrieval in collaborative photo collections," Proc. 2nd Int. Conf. Multimedia Retrieval, ICMR '12, p.21, ACM, 2012.

[10] J.C. Platt, M. Czerwinski, and B.A. Field, "Phototoc: Automatic clustering for browsing personal photographs," Proc. 2003 Joint Conf. Information, Communications and Signal Processing and 4th Pacific Rim Conf. Multimedia, vol.1, pp.6-10, 2003.

[11] M. Naaman, Y.J. Song, A. Paepcke, and H. Garcia-Molina, "Automatic organization for digital photographs with geographic coordinates," Proc. 4th ACM/IEEE-CS Joint Conference on Digital Libraries, JCDL '04, pp.53-62, ACM, 2004.

[12] P. Sinha, "Summarization of archived and shared personal photo collections," Proc. 20th Int. Conf. Companion on World Wide Web, WWW'11, pp.421-426, ACM, 2011.

[13] G. Kim, L. Sigal, and E.P. Xing, "Joint summarization of large-scale collections of web images and videos for storyline reconstruction," Proc. 2014 Conf. Computer Vision and Pattern Recognition, CVPR '14, pp.4225-4232, IEEE, 2014.

[14] M. Cooper, J. Foote, A. Girgensohn, and L. Wilcox, "Temporal event clustering for digital photo collections," Trans. Multimedia Computing, Communications, and Applications, vol.1, no.3, pp.269-288, 2005.

[15] A.C. Loui and A. Savakis, "Automated event clustering and quality screening of consumer pictures for digital albuming," Trans. Multimedia, vol.5, no.3, pp.390-402, 2003.

[16] N. Imran, J. Liu, J. Luo, and M. Shah, "Event recognition from photo collections via pagerank," Proc. 17th Int. Conf. Multimedia, MM '09, pp.621-624, ACM, 2009.

[17] C.L. Wen et al., Event-centric Twitter photo summarization, Ph.D. thesis, Massachusetts Institute of Technology, 2014.

[18] J.M. Zacks and B. Tversky, "Event structure in perception and conception," Psychological bulletin, vol.127, no.1, pp.3-21, 2001.

[19] M. Osborne and M. Dredze, "Facebook, twitter and google plus for breaking news: Is there a winner?," Proc. 8th Int. Conf. Weblogs and Social Media, ICWSM '14, pp.611-614, 2014.

[20] T. Sakaki, M. Okazaki, and Y. Matsuo, "Tweet analysis for realtime event detection and earthquake reporting system development," Trans. Knowledge and Data Engineering, vol.25, no.4, pp.919-931, 2013.

[21] J.M. Zacks, N.K. Speer, K.M. Swallow, T.S. Braver, and J.R. Reynolds, "Event perception: a mind-brain perspective," Psychological bulletin, vol.133, no.2, pp.273-293, 2007.

[22] R. Agrawal, S. Gollapudi, A. Halverson, and S. Ieong, "Diversifying search results," Proc. 2nd Int. Conf. Web Search and Data Mining, WSDM '09, pp.5-14, ACM, 2009

[23] K. Beyer, J. Goldstein, R. Ramakrishnan, and U. Shaft, "When is "nearest neighbor" meaningful?," Proc. 7th Int. Conf. Database Theory, ICDT '99, pp.217-235, Springer-Verlag, 1999.

[24] G.L. Nemhauser, L.A. Wolsey, and M.L. Fisher, "An analysis of approximations for maximizing submodular set functions-i," Mathematical Programming, vol.14, no.1, pp.265-294, 1978.

[25] G. Salton and M.J. McGill, Introduction to modern information retrieval, McGraw-Hill, 1986.

[26] C. Silberer, V. Ferrari, and M. Lapata, "Models of semantic representation with visual attributes," Proc. 51st Ann. Meeting of the Association for Computational Linguistics, ACL '13, pp.572-582, 2013.
[27] A. Makadia, V. Pavlovic, and S. Kumar, "A new baseline for image annotation," Proc. 10th European Conf. Computer Vision, ECCV '08, pp.316-329, 2008.

[28] G. Jeh and J. Widom, "Simrank: a measure of structural-context similarity," Proc. 8th Int. Conf. Knowledge Discovery and Data Mining, KDD '02, pp.538-543, ACM, 2002.

[29] J. Carbonell and J. Goldstein, "The use of mmr, diversity-based reranking for reordering documents and producing summaries," Proc. 21st Ann. Int. Conf. Research and Development in Information Retrieval, SIGIR '98, pp.335-336, 1998.

[30] T. Pedersen, S. Patwardhan, and J. Michelizzi, "Wordnet::similarity: measuring the relatedness of concepts," HLT-NAACL: Demonstration Papers, pp.38-41, Association for Computational Linguistics, 2004.

[31] Y. Jing and S. Baluja, "Visualrank: applying pagerank to large-scale image search," Trans. Pattern Analysis and Machine Intelligence, vol.30, no.11, pp.1877-1890, Nov 2008.

[32] A. Krause and C. Guestrin, "Near-optimal observation selection using submodular functions," Proc. 22nd Nat. Conf. Artificial Intelligence, AAAI'07, pp.1650-1654, 2007.

\section{Appendix: Proof for Section 4.6}

As stated, Eq. (5) is NP-hard, which prevents us from finding the best photo set that can maximize our function. Fortunately, this function is rich in structure, enables a greedy algorithm to be applied, and solves the problem by finding a good approximation to the optimum. We prove the submodularity and monotonicity of the objective function that admits the greedy algorithm.

\section{A.1 Proof of Submodularity}

According to [24], submodularity can be defined as follows.

Definition 10 (Submodularity): Given a finite ground set $N$, a set function $2^{N} \rightarrow \mathbb{R}$ is submodular if and only if for all sets $S, T \subset N$ such that $S \subset T$, and $d \in N \backslash T$, $f(S \cup\{d\})-f(S) \geqslant f(T \cup\{d\})-f(T)$.

Lemma 2: $f(S, e)$ is a submodular function.

Proof 1: Intuitively, the objective function is submodular because a photo set would have already conveyed an event to the user, and therefore, the incremental gain for an additional photo is smaller. Let us now prove the submodularity mathematically.

The class of submodular functions is closed under nonnegative linear combinations based on the property of submodularity [32]. Three criteria in the function are all nonnegative; therefore, to prove the submodularity of function $f$, we only need to prove the submodularity of each function individually. As proved in [22], functions $\operatorname{SubCov}(S, e)$ and $\operatorname{SupCov}(S, e)$ are submodular. Let $S, T$ be two arbitrary sets of photos related by $S \subset T$. Let $m$ be a photo not in T. $S^{\prime}$ denotes $S \cup\{m\}$, and $T^{\prime}$ for $T \cup\{m\}$.

$$
\begin{aligned}
& \operatorname{SibDif}\left(S^{\prime}, e\right)-\operatorname{SibDif}(S, e) \\
& =\prod_{s \in S} \max _{v \in \operatorname{Sib}(e)} P(c=1 \mid s, v) \cdot(1-\max P(c=1 \mid m, v))
\end{aligned}
$$


Similarly, the following formula is workable:

$$
\begin{aligned}
& \operatorname{SibDif}\left(T^{\prime}, e\right)-\operatorname{SibDif}(T, e) \\
& =\prod_{s \in T} \max _{v \in \operatorname{Sib}(e)} P(c=1 \mid s, v) \cdot \prod_{s \in T \backslash S} \max _{v \in \operatorname{Sib}(e)} P(c=1 \mid s, v) \\
& \cdot(1-\max P(c=1 \mid m, v))
\end{aligned}
$$

For each photo $s$ and an event $v, P(c=1 \mid s, v)$ is the probability that $s$ covers $v$, which means $P(c=1 \mid s, v)$ has a value between 0 and 1 . Thus,

$$
\prod_{s \in T \backslash S} \max _{v \in \operatorname{Sib}(e)} P(c=1 \mid s, v) \leqslant 1
$$

Therefore, we conclude that

$$
\operatorname{SibDif}\left(S^{\prime}, e\right)-\operatorname{SibDif}(S, e) \geqslant \operatorname{SibDif}\left(T^{\prime}, e\right)-\operatorname{SibDif}(T, e)
$$

As a result, the function $\operatorname{SibDif}(S, e)$ is submodular, and thus objective function $f(S, e)$ is also submodular.

\section{A.2 Proof of Monotonicity}

In calculus, if a function is monotonically increasing, it should satisfy the following condition:

Definition 11 (Monotonic Increasing): Given a finite ground set $N$, a set function $2^{N} \rightarrow \mathbb{R}$ is monotonic increasing if and only if for all sets $A, B \subset N, A \subset B, f(A) \leqslant f(B)$.

Intuitively, a function is monotonic increasing when the function between ordered sets can preserve the given order. In our case, with more photos in a photo set, the perceptual quality of the photo set is higher.

Lemma 3: $f(S, e)$ is monotonic increasing.

Proof 2: According to the definition of monotonic increasing, a non-negative linear combination of monotonic increasing functions is also monotonic increasing based on the property of adding inequalities (if $a>b$ and $c>d$, then $a+c>d+d$ ). Let us prove each of the three criteria one by one. Let $S, T$ be two arbitrary sets of photos related by $S \subset T$. On the basis of the above demonstration, we can easily get:

$$
\begin{aligned}
& \operatorname{SubCov}(T, e)-\operatorname{SubCov}(S, e) \\
& =\sum_{v \in \operatorname{Sub}(e)} P(v \mid e) \cdot\left(\prod_{s \in S}(1-P(c=1 \mid s, v))\right) \\
& \cdot\left(1-\prod_{s \in T \backslash S}(1-P(c=1 \mid s, v))\right)
\end{aligned}
$$

Since the probability of a photo that covers an event is between 0 and 1 , we can obtain:

$$
\operatorname{SubCov}(T, e)-\operatorname{SubCov}(S, e) \geqslant 0
$$

Thus, $\operatorname{SubCov}(S, e)$ and $\operatorname{SupCov}(S, e)$ are confirmed to be monotonic increasing.
In the case of difference from sibling-events $\operatorname{SibDif}(s, e)$, we can have:

$$
\begin{aligned}
& \operatorname{SibDif}(T, e)-\operatorname{SifDif}(S, e) \\
& =\prod_{s \in T} \max _{v \in \operatorname{Sib}(e)} P(c=1 \mid s, v) \cdot\left(1-\prod_{s \in T \backslash S} \max _{v \in \operatorname{Sib}(e)} P(c=1 \mid s, v)\right)
\end{aligned}
$$

As clarified in the part where we prove submodularity, $P(c=1 \mid s, v)$ is between 0 and 1 for every photo $s$ and event $v$, so the above formula can be deduced to be non-negative, i.e.:

$$
\operatorname{SibDif}(T, e)-\operatorname{SibDif}(S, e) \geqslant 0
$$

Hence, function $\operatorname{SibDif}(s, e)$ is monotonic increasing as desired. We can safely draw the conclusion that $f(S, e)$ is monotonic increasing.

With the satisfaction of submodularity and monotonicity, we eventually confirm that the greedy algorithm is a proper approach to generate an approximate photo set to the optimum photo set.

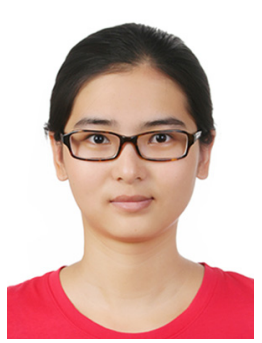

Bei Liu received the B.S. degree from Nanjing University, China in 2011 and the M.S. degree from Kyoto University, Japan in 2014. Since 2014 she has been a Ph.D candidate in Graduate School of Informatics, Kyoto University, Japan. Her research interests include multimedia information retrieval and user cognition in search.

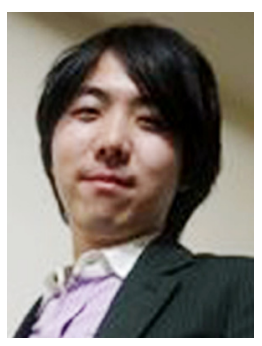

Makoto P. Kato received the B.S., M.S., and $\mathrm{Ph}$.D. degrees from Kyoto University, Japan in 2008, 2009, and 2012, respectively. He is currently an assistant professor at Kyoto University. His research interests include interactive information retrieval, user behavioral analysis in search, and search intent detection.

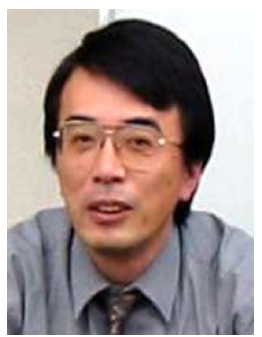

Katsumi Tanaka received the B.S., M.S., and Ph.D. degrees in Information Science from Kyoto University, Japan, in 1974, 1976 and 1981, respectively. In 1986, he joined Department of Instrumentation Engineering, Faculty of Engineering at Kobe University, Japan, as an associate professor. In 1994, he became a full professor in Faculty of Engineering, Kobe University. Since 2001, he has been a professor of Graduate School of Informatics, Kyoto University. His research interests include database theory and systems, web information retrieval, and multimedia retrieval. 